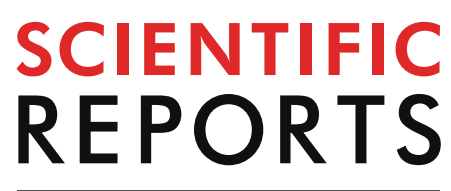

natureresearch

Check for updates

\title{
Polyethylene glycol $(5,000)$ succinate conjugate of lopinavir and its associated toxicity using Danio rerio as a model organism
}

\author{
Oluwole Samuel Aremu ${ }^{1 \bowtie}$, Lebogang Katata-Seru ${ }^{1 \bowtie}$, Zimbili Mkhize ${ }^{1}$, Tarryn Lee Botha ${ }^{2}$ \& \\ Victor Wepener ${ }^{2}$
}

Lopinavir (LPV), a well-known drug administered in human immunodeficiency virus (HIV) infection, has shown limitation for pediatric treatment owing to poor aqueous solubility that gives rise to limited oral bioavailability and short plasma half-life (5-6 h). Polymers such as polyethylene glycol (PEG) have been used as drug carriers to improve their solubility. This study reports the preparation of polyethylene glycol $(5,000)$ succinate (PEG-Suc-LPV) conjugate of LPV by the esterification method. The disappearance of the $3,395 \mathrm{~cm}^{-1}$ (O-H stretch of $\mathrm{COOH}$ ) band for Polyethylene glycol $(5,000)$ succinate (PEG-SUC) confirms the formation ester linkage with the OH group of LPV which is also confirmed by ${ }^{1} \mathrm{H}$ NMR analysis. The XRD for the conjugate showed a broad, amorphous peak while pure PEG, SUc, LPV are crystalline. DSC analysis showed that the conjugate exhibited new broad and diffuse peaks, confirming that they did exist in an amorphous state as multiple complexes. The conjugate showed improved solubility and activity with reduced toxicity compared to pure LPV. The solubility of LPV increased significantly from 80 to $318 \mathrm{ppm}$. Furthermore, an aquatic toxicity test using Danio rerio showed that the conjugate had a lower $\mathrm{LC}_{50}(60.8 \mathrm{ppm})$ when compared to the pure LPV drug LC 50 (6.42 ppm). These results suggest PEG-Suc conjugate of LPV as an efficient carrier for enhanced hydrophilicity and anti-HIV property of LPV.

The advancement of antiretroviral (ARV) therapy has managed to increase the lifespan of people living with human immunodeficiency virus (HIV). Recently, it was reported that 23.3 million people since 2018 were receiving antiretrovirals (ARVs) compared to 21.7 million in $2017^{1}$. However, some of the critical challenges facing HIV treatments includes low aqueous solubility, extensive first-pass metabolism, low bioavailability, and lack of patient adherence. An illustrative example is the orally administered lopinavir (LPV) drug that has shown low bioavailability because of poor aqueous solubility, limited intestinal uptake caused by efflux, and cytochrome $\mathrm{P} 450^{2,3}$. Hence the drug is always co-administered with ritonavir ${ }^{3}$.

Novel nanoformulation approaches to enhance the oral bioavailability of LPV have been investigated by various researchers ${ }^{4-8}$. Patel made an LPV propliposome presenting a vesicle of $659.7 \mathrm{~nm}$ Patel, et al. 4 fabricated LPV propliposome exhibiting a vesicle of $659.7 \mathrm{~nm}$. Their obtained results showed a higher oral bioavailability in rats as compared to pure LPV suspension. The development of pegylated drug conjugates has gained an interest in ARVs ${ }^{9}$. Li synthesized pegylated zidovudine conjugates, and their pharmacokinetic studies showed an increased bioavailability and a 2-threefold prolonged half-life compared to free AZT.

Hence, this study report for the first time the synthesis and characterization of PEG-Suc-LPV. Besides, its solubility and their toxicity studies with Danio rerio, Zebrafish. Zebrafish have been studied extensively and are seen as an internationally accepted model using OECD (eco) toxicology studies based on their close homology with the human genome ${ }^{10}$. Since a significant primary focus of this study based on increasing the solubility of LPV, the use of zebrafish played a complementary role as an appropriate effect-based monitoring tool under the water framework directive ${ }^{11}$.

${ }^{1}$ Chemistry Department, Faculty of Natural and Agricultural Sciences, North-West University, Mmabatho 2735, South Africa. ${ }^{2}$ Water Research Group, Unit for Environmental Sciences and Management, North-West University, Private Bag X6001, Potchefstroom 2520, South Africa. ${ }^{\square}$ email: aremu.oluwole@gmail.com; Lebo.Seru@ nwu.ac.za 


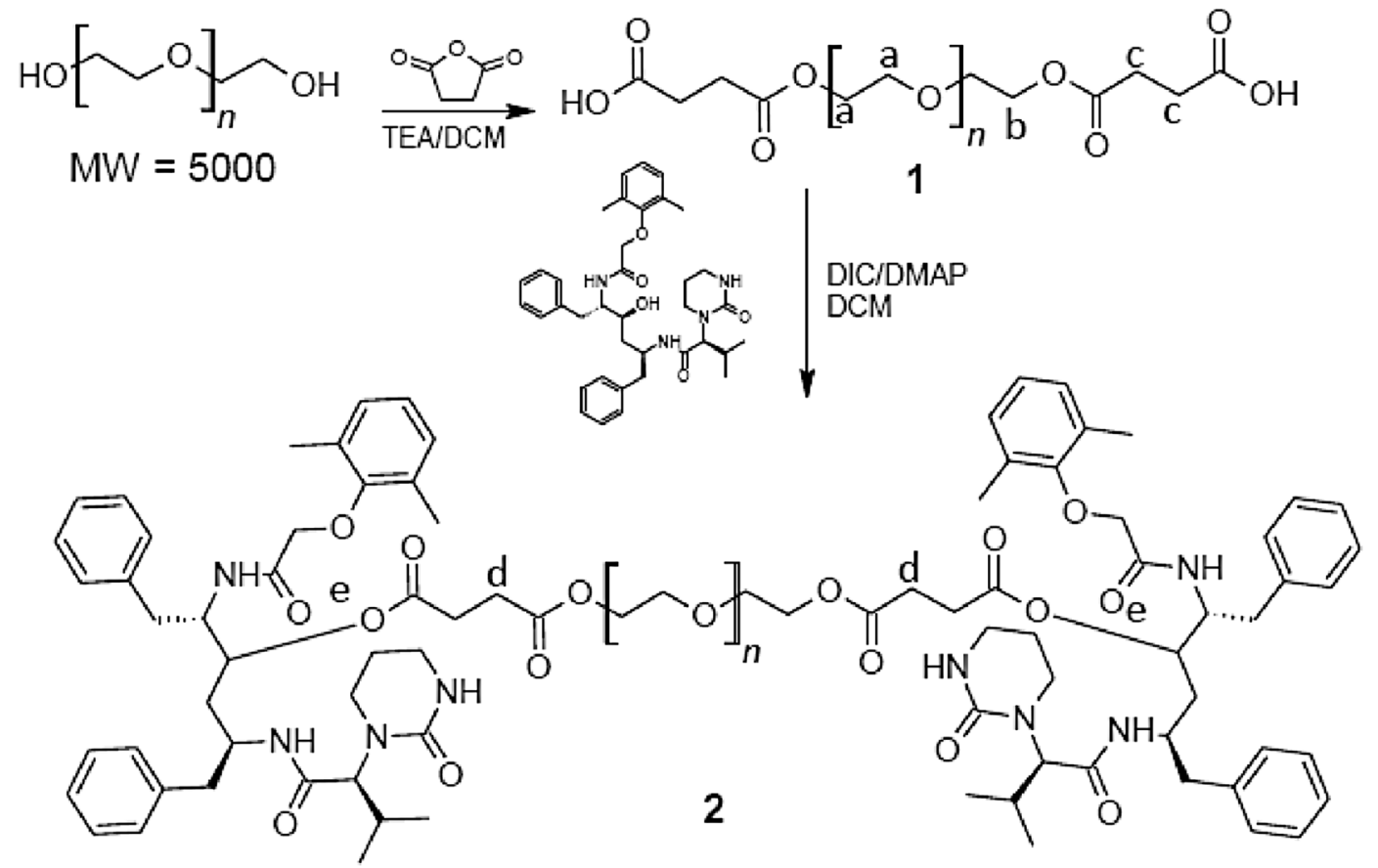

Scheme 1. Synthetic scheme of the succinate derivatives pegylated lopinavir.

\section{Methods}

PEG with two hydroxyl terminals and MW of $5 \mathrm{kDa}$-unit, succinic anhydride (SA), $N, N^{3}$-diisopropyl carbodiimide (DIC), 4-dimethyl aminopyridine (DMAP), triethylamine (TEA), dichloromethane, dimethyl sulfoxide (DMSO), acetonitrile and dimethylformamide (DMF) were all purchased from Sigma Aldrich (South Africa).

Synthesis of PEG-Suc 5 kDa. The synthesis of Pegylated Succinate (PEG-Suc) was accomplished following a modified method based on that of Luo et al. ${ }^{12}$. PEG $5 \mathrm{kDa}(100 \mathrm{mg}, 0.200 \mathrm{mmol}, 1.0$ equiv. $)$ was dissolved in $2 \mathrm{~mL}$ toluene and evaporated three times to remove the water, with the dried PEG dissolved in dichloromethane under nitrogen. The solution cooled to $0{ }^{\circ} \mathrm{C}$, SA was added $(10 \mathrm{mg}, 0.0899 \mathrm{mmol}, 6.0$ equiv.), and TEA $(0.14 \mu \mathrm{L}$, $0.098 \mathrm{mmol}, 6.0$ equiv.) was dropped with a syringe. The reaction was then kept on ice for $60 \mathrm{~min}$ and was stirred at $25^{\circ} \mathrm{C}$ for $24 \mathrm{~h}$. The PEG-Suc was purified by precipitation in cold diethyl ether three times, the precipitates were dried under vacuum at room temperature overnight, and the yield was 95\% at PEG MW (Scheme 1(1)).

Synthesis of PEG-Suc-LPV conjugates 5 kDa. The synthesis of Pegylated-Succinate-Lopinavir conjugate, (PEG-Suc-LPV) was prepared using a modified method based on that of Luo et al. ${ }^{12}$. PEG-Suc, $5 \mathrm{kDa}$ (100 mg, $0.200 \mathrm{mmol}, 1.0$ equiv.), LPV (37 mg, $0.0387 \mathrm{mmol}, 2.5$ equiv.), and DMAP (5 mg, 0.0.397 mmol, 2.5 equiv.) were dissolved in $1.5 \mathrm{~mL}$ DCM under nitrogen, with the solution placed on ice and DIC $(2.0 \mu \mathrm{L}$, $0.0390 \mathrm{mmol}, 2.5$ equiv.) being added dropwise into the flask, the reaction was stirred for $24 \mathrm{~h}$ at room temperature, and the conjugate was purified by precipitation in cold diethyl ether three times and dried under vacuum. (Scheme1 (2)).

Attenuated total reflection Fourier transform infrared (ATR-FTIR) spectroscopy. Cary 600 Series ATR-FTIR spectrometer over the range 4,000-400 $\mathrm{cm}^{-1}$ were used to record the spectra of the conjugates' compatibility to study the interactions between PEG, SA, LPV, PEG-Suc, and PEG-Suc-LPV. The samples were placed directly in the FTIR sample holder in direct contact with the total reflection accessory crystal and infrared spectra in transmittance mode ${ }^{13}$.

Thermal analysis. The sample thermal properties were determined on an indium-calibrated Mettler Toledo differential scanning calorimeter (DSC, Perkin Elmer, Überlingen, Germany), with an initial heating scan of 10 to $100{ }^{\circ} \mathrm{C}$, a cooling scan of 100 to $-10^{\circ} \mathrm{C}$ and another heating scan of -10 to $100^{\circ} \mathrm{C}$ at a scan rate of $10^{\circ} \mathrm{C} /$ min. Crystallinity was assumed to be proportional to the experimental heat of fusion of $139.5 \mathrm{~J} \mathrm{~g} 1$ for the $100 \%$ crystalline samples ${ }^{14}$.

X-ray diffraction (XRD). The X-ray powder diffraction patterns were obtained using a PANalytical Empyrean diffractometer (PANalytical, Almelo, Netherlands), the measurement conditions being: target, $\mathrm{Cu}$; voltage, $40 \mathrm{kV}$; current, $30 \mathrm{~mA}$; divergence slit, $2 \mathrm{~mm}$; anti-scatter slit, $0.6 \mathrm{~mm}$; detector slit, $0.2 \mathrm{~mm}$; monochromator; scanning speed, $2^{\circ} / \mathrm{min}$ (step size, $0.025^{\circ}$; step time, $\left.1.0 \mathrm{~s}\right)^{15}$. 


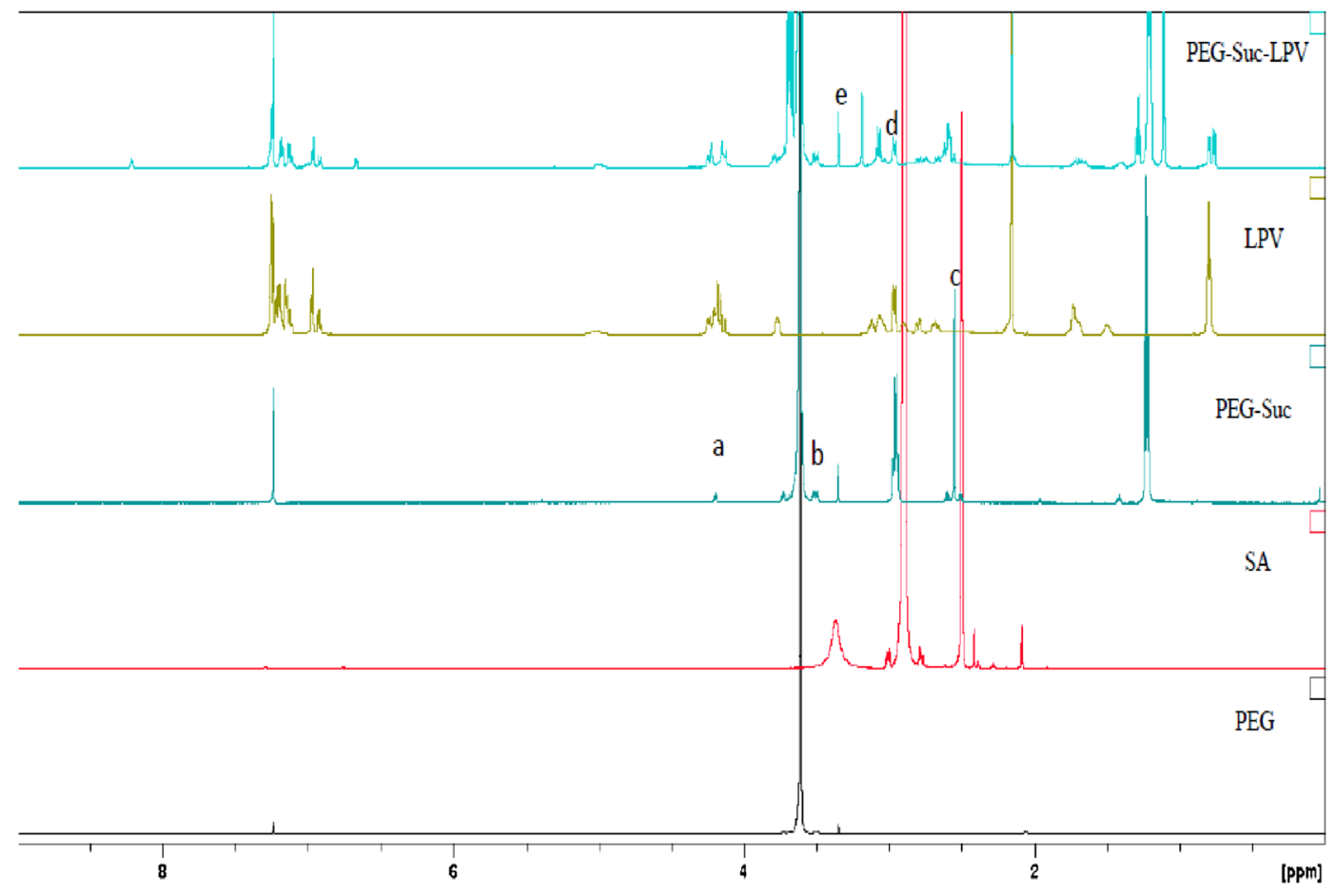

Figure 1. Proton NMR of the PEG-Suc-LPV conjugate and its different starting materials.

Solubility studies. Solubility determination of the conjugates and the drug were added in vials containing distilled water, it was shaken for $24 \mathrm{~h}$ at room temperature in a bath with a shaker until equilibrium is reached. The resultant suspension was then filtered through Whatman filter paper No. 1 and suitably diluted with distilled water. Finally, the filtrate was analyzed by UV. 100 to $900 \mathrm{mg} / \mathrm{ml}$ of the pure drug were prepared, and the absorbances measured at $257 \mathrm{~nm}$ using a Perkin Elmer Lambda $365 \mathrm{UV}$-Visible recording spectrophotometer. A standard curve was plotted $\left(\mathrm{R}^{2}\right.$ 0.9975) using the acquired data from the absorbance of the filtered samples, then solubility was calculated from the values obtained from the standard curve of the pure drug ${ }^{16}$.

Toxicity studies using Danio rerio. All wild type zebrafish used within this study were maintained in a Tecniplast ZebTec system at the National Aquatic Bioassay facility at North-West University, with the juvenile fish used within this study being bred two weeks before testing. The South African National Standards (SANS, 2008: $10,386^{17}$ for the care and use of animals for scientific purposes) was followed for all husbandry and breeding. The fish acute lethality test ${ }^{11}$ was performed on 14 -day old juveniles following OECD protocols, according to ethical procedures as approved by the AnimCare Committee at North-West University (NWU-00269-16-A5).

The standard OECD reconstituted water contained $\mathrm{CaCl}_{2} \cdot 2 \mathrm{H}_{2} \mathrm{O}, \mathrm{MgSO}_{4} .7 \mathrm{H}_{2} \mathrm{O}, \mathrm{NaHCO}_{3}$, and $\mathrm{KCl}$ (Sigma Aldrich, South Africa) and was aerated for $24 \mathrm{~h}$ before use to obtain at least $60 \%$ oxygen saturation. Briefly, three $500 \mathrm{~mL}$ beaker replicates containing seven fish per beaker were set up per concentration tested. Both LPV and PEG-Suc-LPV conjugate were prepared in ultrapure water at $100 \mathrm{mg} / \mathrm{L}$ stock concentrations and sonicated in a bath sonicator filled with $900 \mathrm{~mL}$ of water at $25^{\circ} \mathrm{C}$ for one hour. Exposure concentrations were made up in a reconstituted OECD medium in a range of $0.1-40 \mathrm{mg} / \mathrm{L}$. A $12 \mathrm{~h}$ light and $12 \mathrm{~h}$ dark cycle was maintained for the duration of the test, with the temperature in the room being set to $28^{\circ} \mathrm{C}$.

Physico-chemical parameters were recorded every $24 \mathrm{~h}$, following standard protocols. The temperature, $\mathrm{pH}$, electrical conductivity, and total dissolved solids were measured using a handheld Eutech pH $110 \mathrm{RS} 232 \mathrm{C}$ meter, Eutech CON 110 RS232C conductivity, and TDS meter. Mortality per beaker was recorded at every $24 \mathrm{~h}$ interval and was regarded when there was no visible gill movement. For a test to be valid, the control had to have less than $10 \%$ mortality for its full duration. A dose-response function was obtained, and a lethal concentration (LCx) value was calculated using the regression analysis (linear or non-linear) in Toxrat Professional. The results were presented as an LC10 (where $10 \%$ of the organisms were affected), an LC20 (20\% affected), and LC50 (50\% affected).

\section{Results and discussion}

Characterization of PEG-Suc-LPV Conjugate. Nuclear magnetic resonance. Figure 1 showed the proton NMR of the PEG-Suc-LPV conjugate and its different starting materials. The ${ }^{1} \mathrm{H}$ NMR 7.24 and $1.56 \mathrm{ppm}$ appear for the chemical shift of the solvent peaks of $\mathrm{CDCl}_{3}$, with the proton assignment of the PEG-Suc shown in Fig. 1. Peak "a" (4.19 (t, J=10.0 Hz, 4H, PEG-O-CH $\left.-\mathrm{CH}_{2}-\mathrm{O}-\mathrm{SA}\right)$, "b" (3.45-3.53 m, PEG backbone), and "c" (2.60 ppm m, 8H, PEG-COO- $\mathrm{CH}_{2}-\mathrm{CH}_{2}-\mathrm{COOH}$ ) indicate conjugation of an ester bond between PEG and SA. In the next step, the PEG-Suc conjugated to LPV via the formation of ester bonds, was also confirmed by ${ }^{1} \mathrm{H}$ NMR. 


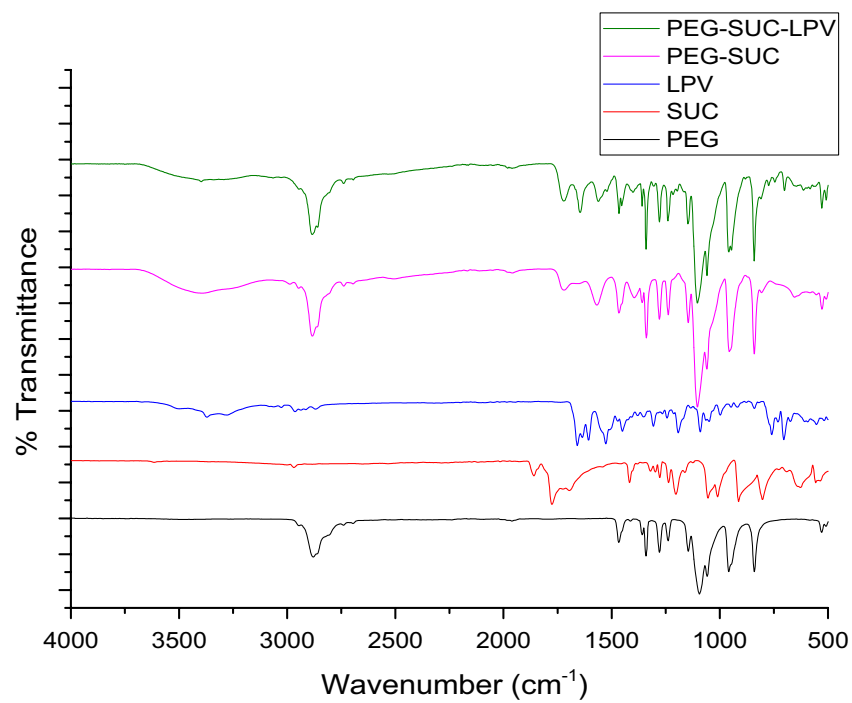

Figure 2. ATR-FTIR of PEG, Suc. PEG-Suc, LPV and PEG-Suc-LPV.

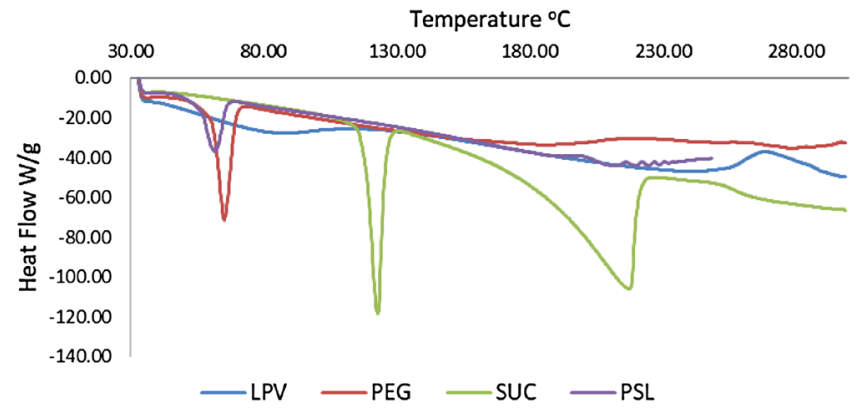

Figure 3. DSC thermograms of PEG, Suc, LPV and PEG-Suc-LPV.

Peak "e" shows the ${ }^{1} \mathrm{H}$ NMR spectra of PEG-Suc-LPV, which exhibited the characteristic peaks of PEG and LPV. The ${ }^{1} \mathrm{H}$ NMR results indicated that the C-3 hydroxyl group of LPV underwent esterification. Compared to the LPV alone (peak "d"), covalent conjugation to the PEG with ester bonds of several different Anti-HIV drugs has been reported to confer improved antiretroviral efficacy and decreased toxicity ${ }^{18}$.

ATR-FTIR. The FTIR spectra of PEG, SA, LPV, PEG-Suc, and PEG-Suc-LPV conjugates shown in Fig. 2, with the characteristic peaks assignments of PEG being $3,411 \mathrm{~cm}^{-1}\left(\mathrm{O} \mathrm{H}\right.$ stretch), $2,877(\mathrm{CH}), 1,466 \mathrm{~cm}^{-1}\left(\mathrm{CH}_{2}\right.$ vibrational stretching for methylene group). The IR spectral bands of SA are $3,616 \mathrm{~cm}^{-1}$ (O H stretch), $2,972 \mathrm{~cm}^{-1}$ $(\mathrm{CH}), 1722 \mathrm{~cm}^{-1}(\mathrm{C}=\mathrm{O})$. The IR spectral band of PEG-Suc: $3,395 \mathrm{~cm}^{-1}(\mathrm{O} \mathrm{H}$ stretch of COOH$), 2,884 \mathrm{~cm}^{-1}$ $(\mathrm{CH}), 1718 \mathrm{~cm}^{-1}\left(\mathrm{C}=\mathrm{O}\right.$ stretching of ester), $1567 \mathrm{~cm}^{-1}$ (C O stretch of $\left.\mathrm{COOH}\right)$. The appearance of COOH confirms that the PEG successfully reacted with $\mathrm{SA}$.

The PEG-Suc-LPV conjugates revealed characteristic absorption peaks at 3,397 cm-1 (NH vibrational peak), 2,884-2,739 $\mathrm{cm}^{-1}$ (C-H stretch of PEG chain), which were stronger and sharper than the LPV and PEG-Suc. The peaks at $1721-1645 \mathrm{~cm}^{-1}$ (C-O stretching of ester), and 1,147-1,103 $\mathrm{cm}^{-1}$ (bridge $\mathrm{C}-\mathrm{O}-\mathrm{C}$ stretch) also found in these spectra, but peaks at $3,395 \mathrm{~cm}^{-1}(\mathrm{O}-\mathrm{H}$ stretch of $\mathrm{COOH})$ and $1718 \mathrm{~cm}^{-1}(\mathrm{C}-\mathrm{O}$ stretch of $\mathrm{COOH})$, which were observed in Fig. 2, both peaks disappeared, indicating the COOH of PEG-Suc had conjugated with the OH of LPV. The above FTIR analysis clearly showed that PEG-Suc-LPV successfully synthesized and it is expected that PEG conjugates will act as prodrugs and dissolution of polyethylene glycol moieties leads to release of parent drug 1 LPV in body fluids without effecting required therapeutic effect. the result above being like that reported by Hifumi et al. ${ }^{13,19}$.

Differential scanning calorimetry. In the DSC, the samples heated at a constant rate of $10{ }^{\circ} \mathrm{C}$ and the amount of energy required detected. The pure LPV thermogram showed an endothermic peak at $88.4^{\circ} \mathrm{C}$, which indicates the crystalline nature of LPV. The PEG-Suc-LPV conjugate thermogram showed an endothermic peak at $60.7^{\circ} \mathrm{C}$ but not at $88.4^{\circ} \mathrm{C}$. Crystallinity reduction occurs after the decline in thermal temperature and melting point. When the crystallinity decreases, the crystalline nature was converted to amorphous structures, which resulted in solubility improvement (Fig. 3$)^{20}$. 


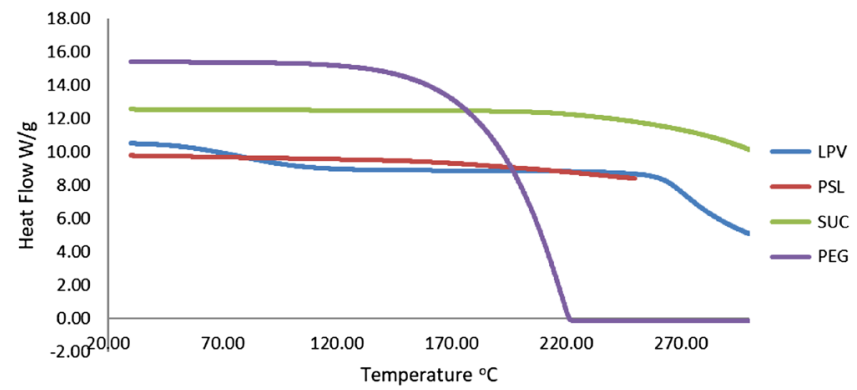

Figure 4. TGA thermograms of PEG, Suc, LPV, and PEG-Suc-LPV.

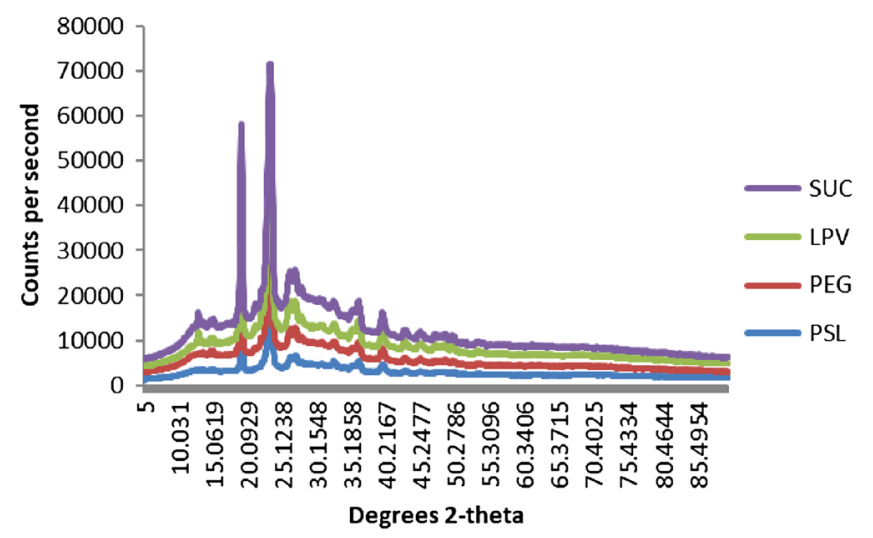

Figure 5. XRD image of PEG, Suc. LPV and PEG-Suc-LPV.

\begin{tabular}{|l|l|l|l|l|}
\hline Batch & Water & $\mathbf{0 . 1 N H C l} \mathbf{( ( 1 . 2 )}$ & PBS (PH 7.4) & MEOH \\
\hline LPV & 80 & 847 & 626 & 555 \\
\hline Conjugate & 318 & 2,231 & 2,531 & 523 \\
\hline
\end{tabular}

Table 1. Saturation Solubility studies of LPV and conjugate (ppm).

Thermogravimetric analysis. As shown in Fig. 4, the TGA thermograms of pure LPV showed a two-step weight loss at $80^{\circ} \mathrm{C}$ and decomposition at $268^{\circ} \mathrm{C}$ compared to the PEG-Suc-LPV, which showed heat stability until $248{ }^{\circ} \mathrm{C}$. This is due to the amorphous phase of the PEG-Suc-LPV compared to the crystallinity phase of the pure LPV, this further confirming the improvement of the solubility of LPV. The PEG showed a complete decomposition between $155-180^{\circ} \mathrm{C}$ and a complete degradation at $220^{\circ} \mathrm{C}$, while the Suc showed one step of weight loss at $280^{\circ} \mathrm{C}$ (Fig. 4$)^{21}$.

X-ray diffraction. To ascertain the physical state and crystallinity of LPV in the polymeric conjugate, the XRD pattern of pure PEG, Suc, LPV, and PEG-Suc-LPV conjugate are presented in Fig. 5. As can be seen, pure PEG, Suc, LPV are highly crystalline and showed several diffraction peaks at $2 \Theta=15.20,21.3,28.13,29.68,3052$, $39.12,40.31 \mathrm{deg}$, while the diffraction peaks of conjugate were not recorded at the same position, which showed less diffraction peaks with low reduction in crystalline peaks. Formation of new peaks in the conjugate might be attributed to a polymorph structure transformation due to the conjugation of PEG, Suc, and LPV. The conjugate showed a broad amorphous peak. This corresponds to the improved solubility of the conjugate ${ }^{22}$.

Solubility studies. The results of the solubility studies are shown in Table 1. It was examined in four different media: aqueous, $0.1 \mathrm{~N} \mathrm{HCl} \mathrm{(1.2),} \mathrm{PBS} \mathrm{(7.4),} \mathrm{and} \mathrm{MeOH.} \mathrm{As} \mathrm{indicated,} \mathrm{pure} \mathrm{LPV} \mathrm{shows} \mathrm{lower} \mathrm{solubility} \mathrm{of}$ $80 \mathrm{ppm}$ in water compared to solubility in other media with 847, 626, 555 (ppm). In contrast, PEG-Suc-LPV showed an improved solubility of sixfold in water, threefold in $0.1 \mathrm{~N}$ of $\mathrm{HCl}$, fourfold PBS, and tenfold in $\mathrm{MEOH}$ compared to free LPV. This enhanced solubility can be attributed to the effect of the formation of amorphous conjugate $^{23}$. 


\begin{tabular}{|l|l|l|l|}
\hline & LC10 & LC20 & LC50 \\
\hline LPV \\
\hline Value [ppm] & n.d & 0.050 & 6.417 \\
\hline Lower 95\%-cl & n.d & n.d & 2.038 \\
\hline Upper 95\%-cl & n.d & 0.302 & 49.157 \\
\hline & LC10 & LC20 & LC50 \\
\hline Conjugate & & & \\
\hline Value [ppm] & 13.357 & 22.473 & 60.804 \\
\hline Lower 95\%-cl & 5.729 & 14.009 & 35.821 \\
\hline Upper 95\%-cl & 20.305 & 39.592 & 307.323 \\
\hline
\end{tabular}

Table 2. Median lethal concentrations for 10, 20 and 50\% (LC10, LC20, and LC50) effect and the corresponding 95\% confidence intervals for test organisms exposed LPV conjugate.

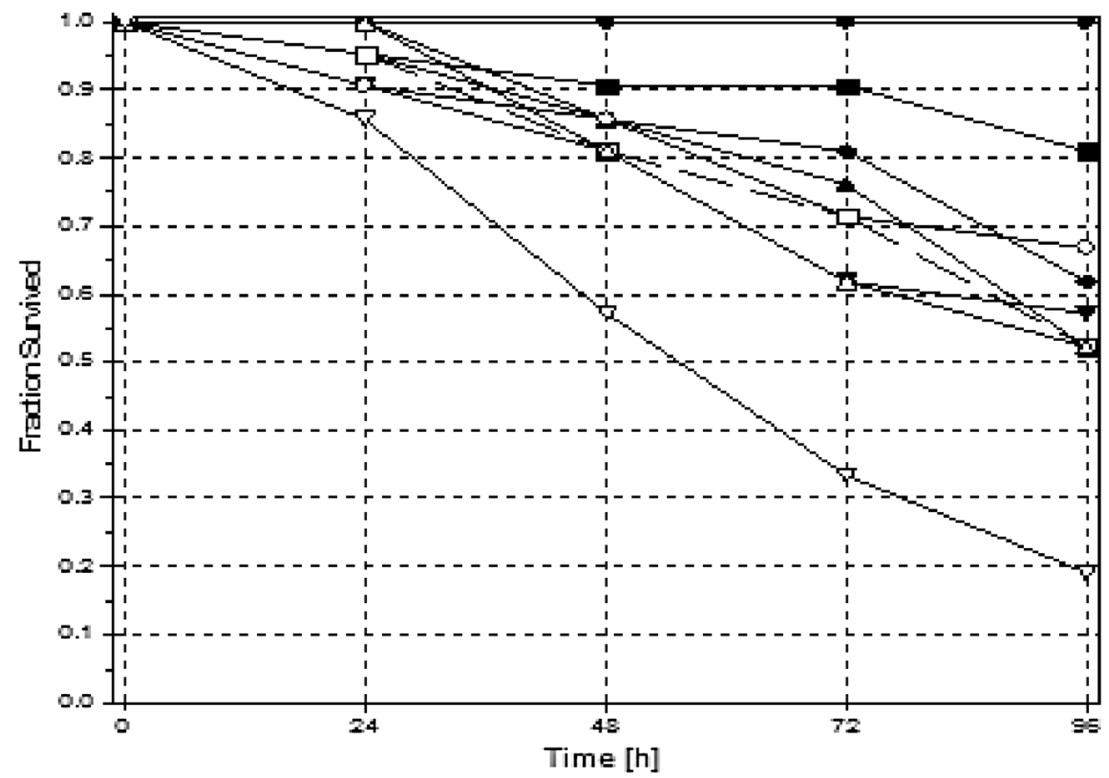

$*$ Control
$-0.100 \mathrm{mg} / \mathrm{L}$
$+0.500 \mathrm{mg} / \mathrm{L}$
$+1.000 \mathrm{mg} / \mathrm{L}$
$+2.000 \mathrm{mg} / \mathrm{L}$
$5.000 \mathrm{mg} / \mathrm{L}$
$\square 10.000 \mathrm{mg} / \mathrm{L}$
$\leftarrow 20.000 \mathrm{mg} / \mathrm{L}$
$\approx 40.000 \mathrm{mg} / \mathrm{L}$

Figure 6. The survival of the introduced 14-day old Danio rerio as observed over a 96-h exposure to varying concentrations of LPV.
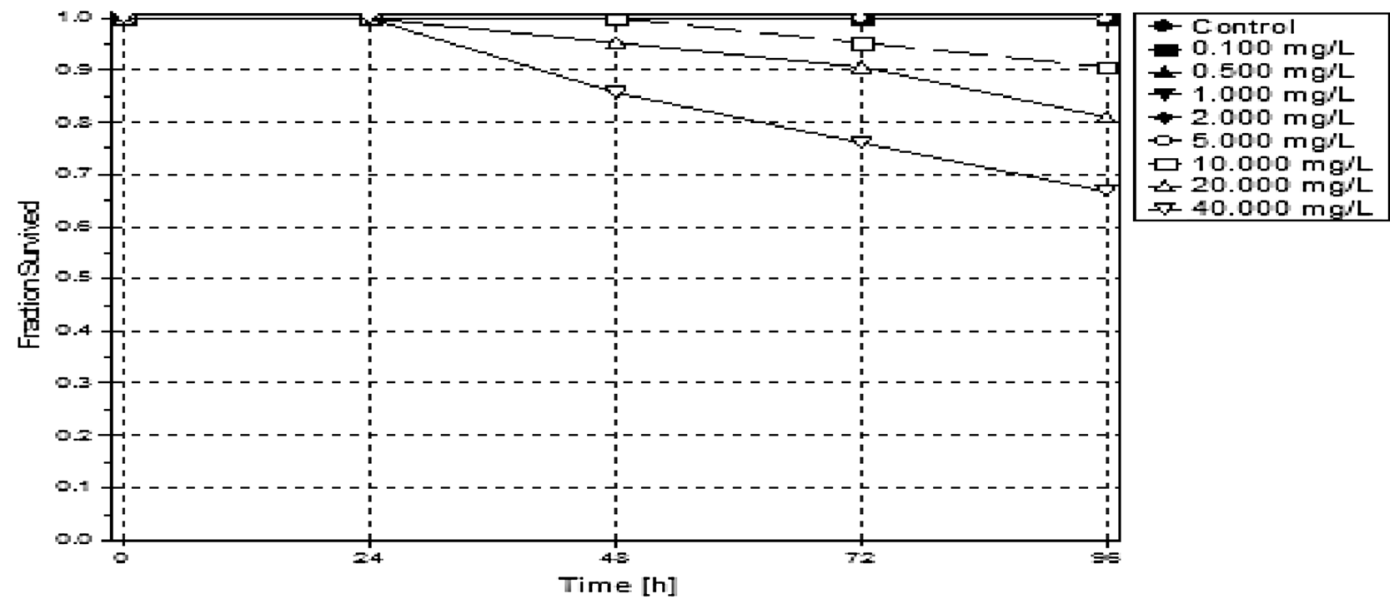

Figure 7. The survival of the introduced 14-day old Danio rerio as observed over a 96-h exposure to varying concentrations of conjugate. 


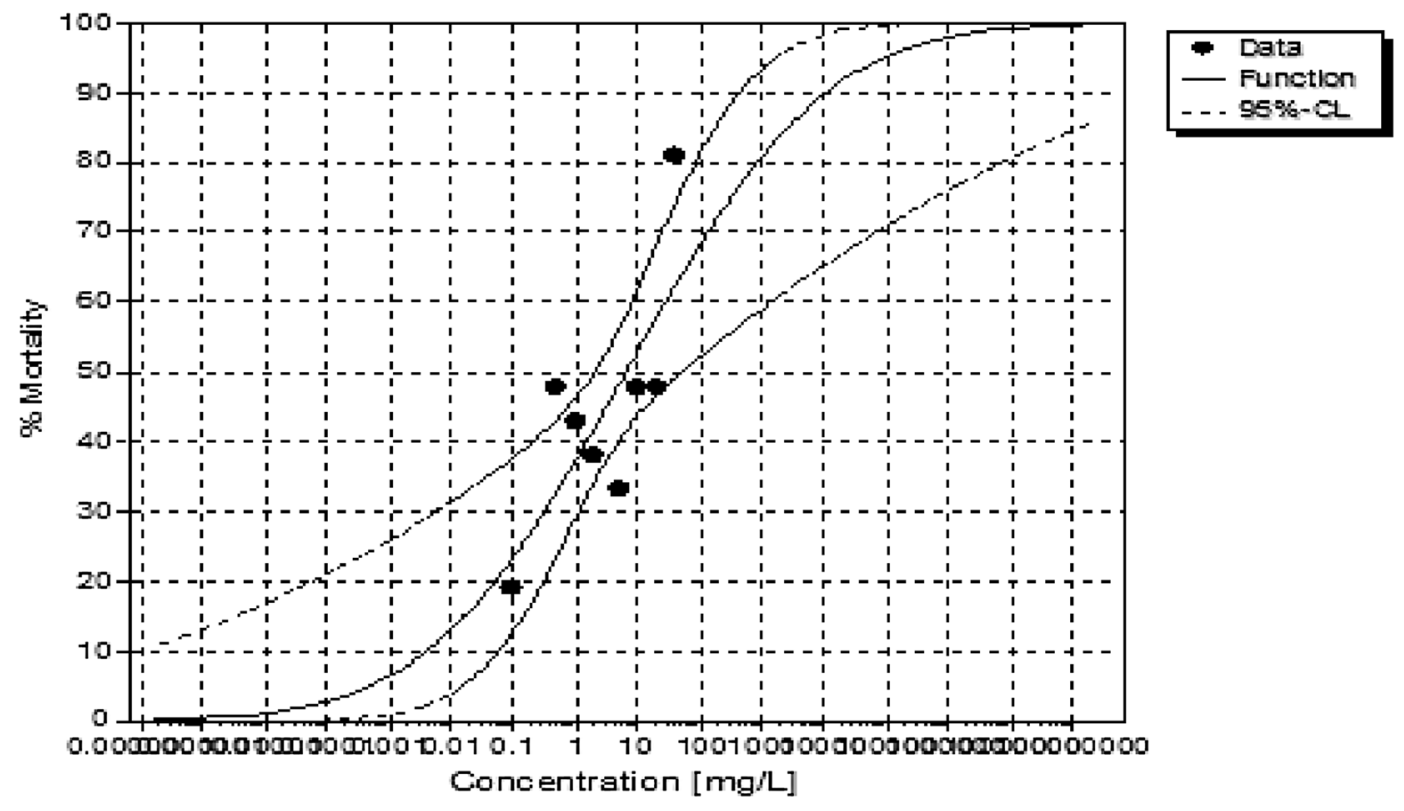

Figure 8. The concentration-effect curve used to calculate LCx values showing the influence of varying concentrations of LPV on Danio rerio.

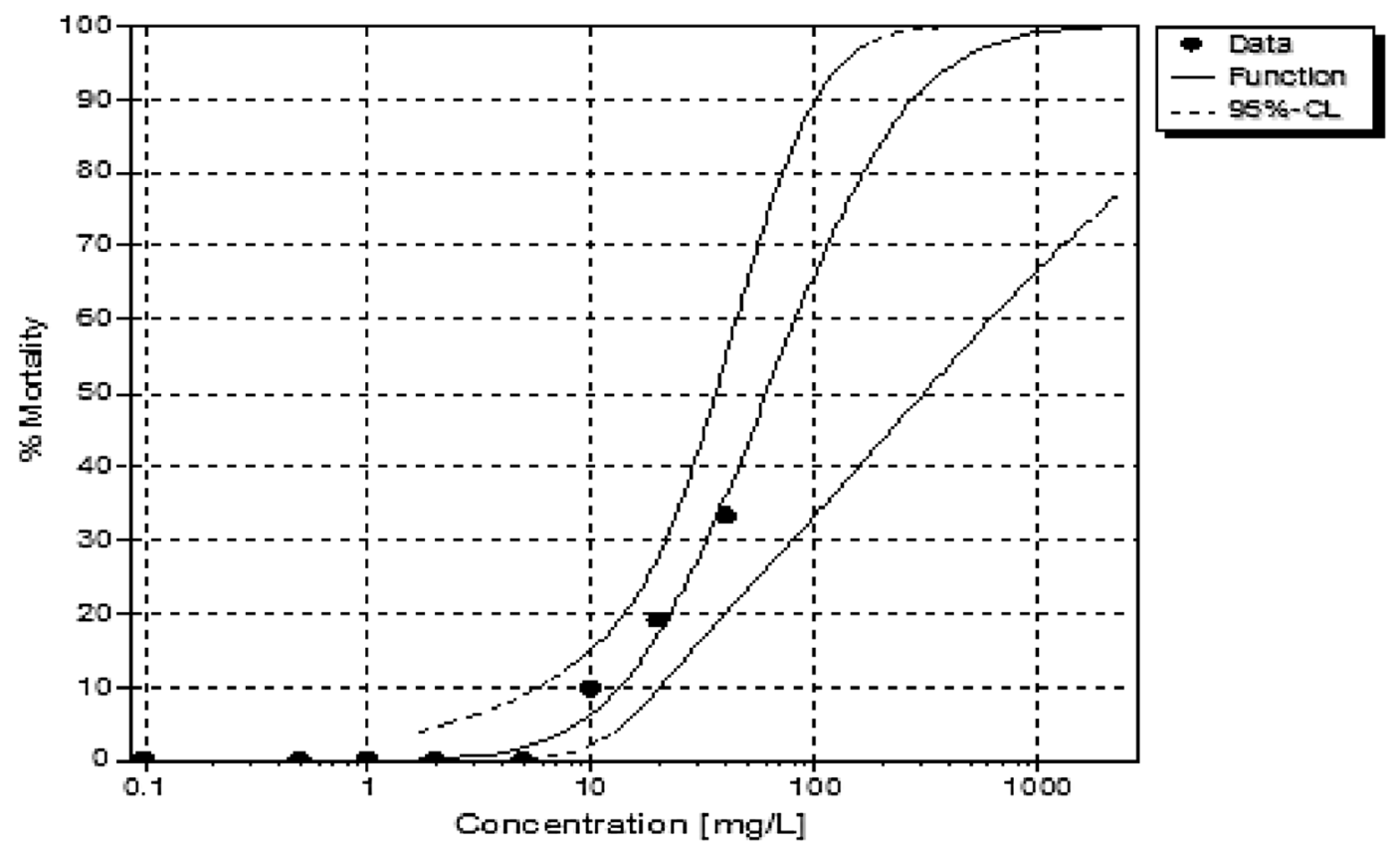

Figure 9. The concentration-effect curve used to calculate LCx values showing the influence of varying concentrations of the conjugate on Danio rerio.

Toxicity studies using Danio rerio. The reconstituted water was maintained at a constant temperature across all test beakers $\left(26.9 \pm 0.16^{\circ} \mathrm{C}\right)$, and the $\mathrm{pH}$ remained in a close range $(7.93 \pm 0.05)$ for the duration of the test. Although, the control and LPV showed a similar range of total dissolved solids (with $841.3 \pm 32.8$, ppm and $820.7 \pm 13.2 \mathrm{ppm}$ respectively), a difference was seen with, the conjugate having a lower value of $536.5 \pm 11.7 \mathrm{ppm}$. The conductivity followed the same pattern, where the control was $1,202.2 \pm 40.8 \mu \mathrm{S}$, LPV $1,184.2 \pm 22.9 \mu \mathrm{S}$ and the conjugate $536.5 \pm 11.7 \mu \mathrm{S}$. This phenomenon can be further related to the solubility seen in different media (Table 1) which shows that as the conjugate reacts with the salts within the reconstituted water, the solubility of the conjugate increases and the salts become unavailable, resulting in a lower conductivity reading within the water medium. 
There was a lower solubility observed during exposures of the LPV and the exposures sedimented out of solution from $20 \mathrm{ppm}$ in the test beaker within $24 \mathrm{~h}$. Methanol was not used as a dispersant in this study to maintain the integrity of the conjugate tested, and all other procedures were applied to both chemicals tested. The LPV had a tenfold higher LC50 (6.417 ppm) than the conjugate form (60.804 ppm) (Table 2). A dose-dependent toxicity was seen within the first $24 \mathrm{~h}$ of exposure. In comparison, the conjugate had lower toxicity observed after $48 \mathrm{~h}$ of exposure (Figs. 6,7).

The concentration-effect curves showed varied toxicity at $96 \mathrm{~h}$ in LPV, which can also be attributed to the low solubility, an effect that was not observed in the conjugate group (Figs. 8, 9). This is one of the first studies reporting acute toxicity of LPV and an LPV conjugate ARV using 14-day old Danio rerio.

\section{Conclusions}

The study managed to synthesize the PEG-Suc-LPV conjugate successfully by using an esterification method, which indicates that this preparation method is a promising approach for establishing the solubility of LPV. The study further confirms that this increased solubility decreased the toxicity of LPV when used in a conjugate form after exposure to the Danio rerio Zebrafish assay.

Received: 30 September 2019; Accepted: 29 June 2020

Published online: 16 July 2020

\section{References}

1. Global HIV \& AIDS Statistics. 2018 Fact sheet. UNAIDS. Available online: https://www.unaids.org/en/resources/fact-sheet (accessed on 14 January 2019).

2. Sharma, P. \& Garg, S. Pure drug and polymer based nanotechnologies for the improved solubility, stability, bioavailability and targeting of anti-HIV drugs. Adv. Drug Deliv. Rev. 62, 491-502 (2010).

3. Panel on antiretroviral therapy and medical management of children living with HIV. (Guidelines for the use of antiretroviral agents in paediatric HIV infection. Available at https://aidsinfo.nih.gov/contentfiles/lvguidelines/pediatricguidelines.pdf. (Accessed 25 May 2018)).

4. Patel, G. M., Shelat, P. K. \& Lalwani, A. N. QbD based development of proliposome of lopinavir for improved oral bioavailability. Eur. J. Pharm. Sci. 108, 50-61 (2017).

5. Fakhar ud, D. \& Khan, G. M. Development and characterisation of levosulpiride-loaded suppositories with improved bioavailability in vivo. Pharm. Dev. Technol. 24, 63-69. https://doi.org/10.1080/10837450.2017.1419256 (2019).

6. Jain, S., Sharma, J. M., Jain, A. K. \& Mahajan, R. R. Surface-stabilized lopinavir nanoparticles enhance oral bioavailability without coadministration of ritonavir. Nanomedicine 8, 1639-1655 (2013).

7. Patel, G., Shelat, P. \& Lalwani, A. Statistical modeling, optimization and characterization of solid self-nanoemulsifying drug delivery system of lopinavir using design of experiment. Drug Del. 23, 3027-3042 (2016).

8. Ravi, P. R., Vats, R., Dalal, V. \& Gadekar, N. Design, optimization and evaluation of poly-e-caprolactone (PCL) based polymeric nanoparticles for oral delivery of lopinavir. Drug Dev. Ind. Pharm. 41, 131-140 (2015).

9. Li, W. et al. Synthesis, In Vitro and In Vivo Release Kinetics, and Anti-HIV Activity of A Sustained-Release Prodrug (mPEG-AZT) of 3'-Azido-3'-deoxythymidine (AZT, Zidovudine). ChemMedChem 5, 1893-1898 (2010).

10. Zhao, H. et al. 20-O-acylcamptothecin derivatives: evidence for lactone stabilization. J. Org. Chem. 65, 4601-4606 (2000)

11. Lammer, E. et al. Is the fish embryo toxicity test (FET) with the zebrafish (Danio rerio) a potential alternative for the fish acute toxicity test?. Comp. Biochem. Physiol. C: Toxicol. Pharmacol. 149, 196-209 (2009).

12. Luo, T. et al. Synthesis and in vitro evaluation of polyethylene glycol-paclitaxel conjugates for lung cancer Therapy. Pharm. Res. 33, 1671-1681 (2016).

13. Ewing, A. V., Clarke, G. S. \& Kazarian, S. G. Attenuated total reflection-Fourier transform infrared spectroscopic imaging of pharmaceuticals in microfluidic devices. Biomicrofluidics 10, 024125 (2016).

14. Castilla-Cortázar, I., Vidaurre, A., Marí, B. \& Campillo-Fernández, A. J. Morphology, crystallinity, and molecular weight of poly ( $\varepsilon$-caprolactone)/graphene oxide hybrids. Polymers 11, 1099 (2019).

15. León-Reina, L. et al. Accuracy in Rietveld quantitative phase analysis: a comparative study of strictly monochromatic $\mathrm{Mo}$ and $\mathrm{Cu}$ radiations. J. Appl. Crystallogr. 49, 722-735 (2016).

16. Hun, L. T. et al. Investigations into the physicochemical, biochemical and antibacterial properties of Edible Bird's Nest. J. Chem. Pharm. Res. 7, 228-247 (2015).

17. Mohr, B. The current status of laboratory animal ethics in South Africa. Altern. Lab. Anim. 41, P48-P51 (2013).

18. Palombo, M., Singh, Y. \& Sinko, P. Prodrug and conjugate drug delivery strategies for improving HIV/AIDS therapy. J. Drug Deliv. Sci. Technol. 19, 3-14 (2009).

19. Hifumi, H., Ewing, A. V. \& Kazarian, S. G. ATR-FTIR spectroscopic imaging to study the drying and dissolution of pharmaceutical polymer-based films. Int. J. Pharm. 515, 57-68 (2016).

20. Rask, M. B., Knopp, M. M., Olesen, N. E., Holm, R. \& Rades, T. Comparison of two DSC-based methods to predict drug-polymer solubility. Int. J. Pharm. 540, 98-105 (2018).

21. Alonzo, D. E., Zhang, G. G., Zhou, D., Gao, Y. \& Taylor, L. S. Understanding the behavior of amorphous pharmaceutical systems during dissolution. Pharm. Res. 27, 608-618 (2010).

22. Anwar, M. et al. Enhanced bioavailability of nano-sized chitosan-atorvastatin conjugate after oral administration to rats. Eur. J. Pharm. Sci. 44, 241-249 (2011).

23. Mandal, A., Pal, D. \& Mitra, A. K. Circumvention of P-gp and MRP2 mediated efflux of lopinavir by a histidine based dipeptide prodrug. Int. J. Pharm. 512, 49-60 (2016).

\section{Acknowledgements}

We would like to send our special gratitude to the Chemistry Department at North-West University (Mafikeng Campus) and Water Research Group Unit for Environmental Sciences and Management, North-West University (Potchefstroom Campus) for providing the research work facility, Sasol Foundation and National Research Foundation in South Africa for their financial support. This research was funded by Sasol Inzalo Foundation and National Research Foundation South Africa, grant number (NRF Thuthuka UID 87987, NRF UID: 106379, NNEP UID: 99024). Opinions, findings, conclusions and recommendations expressed in this publication are that of the authors, and the NRF accepts no liability whatsoever in this regard. This is contribution number 469 of the North-West University Water Research Group. 


\section{Author contributions}

Conceptualization, L.K., and Z.M.; methodology, O.S.A., and T.L.B; software, O.S.A., and T.L.B.; validation, L.K., V.W. and O.S.A.; formal analysis, O.S.A., and T.L.B; investigation, O.S.A., and T.L.B.; resources, L.K., V.W. and O.S.A.; data curation, L.K., V.W., and O.S.A.; writing-original draft preparation, O.S.A., and T.L.B.; writingreview and editing, L.K., V.W., Z.M., T.L.B., and O.S.A.; visualization, V.W., L.K., V.W., Z.M., T.L.B. and O.S.A; supervision, L.K.; project administration, L.K.; funding acquisition, L.K., V.W and O.S.A.

\section{Competing interests}

The authors declare no competing interests.

\section{Additional information}

Correspondence and requests for materials should be addressed to O.S.A. or L.K.-S.

Reprints and permissions information is available at www.nature.com/reprints.

Publisher's note Springer Nature remains neutral with regard to jurisdictional claims in published maps and institutional affiliations.

Open Access This article is licensed under a Creative Commons Attribution 4.0 International

License, which permits use, sharing, adaptation, distribution and reproduction in any medium or format, as long as you give appropriate credit to the original author(s) and the source, provide a link to the Creative Commons license, and indicate if changes were made. The images or other third party material in this article are included in the article's Creative Commons license, unless indicated otherwise in a credit line to the material. If material is not included in the article's Creative Commons license and your intended use is not permitted by statutory regulation or exceeds the permitted use, you will need to obtain permission directly from the copyright holder. To view a copy of this license, visit http://creativecommons.org/licenses/by/4.0/.

(c) The Author(s) 2020 